\title{
Policy Based Management of Wireless Networks
}

\author{
R. State, G. Vivier \\ Motorola Labs \\ Espace technologique, Commune de St Aubin, \\ 91193 Gif-sur-Yvette Cedex, France \\ tel. : $+33-(0) 1-69352500$ \\ [state,vivier]@crm.mot.com
}

\begin{abstract}
The advent of multi-technologies networks offering ubiquitous services over advanced network infrastructure demands an integrated management approach. We address in this paper a policy based management approach for end to end services offered over reconfigurable radio architectures. We show how to integrate our solution into the larger context of directory enabled networks, thus allowing the management of radio resources in the framework of the supported IP based network.
\end{abstract}

Key words: Service management, DEN, SDR, policy based management

\section{INTRODUCTION}

A new communication model, often named Beyond $3 \mathrm{G}$ (B3G), is rapidly emerging as potential solution towards an open, smooth and flexible integration of different access technologies such as cellular, broadcast or Wireless LAN, within the larger context of end to end IP connectivity. The different radio technologies will be integrated in an expandable manner, to complement each other, providing an efficient use of the scarce radio spectrum and above all, a wide range of services to the final user. On the terminal side, it is expected with the progress of Software Definable Radio (SDR) that a terminal would be able to configure itself from one radio technology to the other in a seamless manner. Future wireless networks will therefore provide added value by allowing to deploy a large variety of

The original version of this chapter was revised: The copyright line was incorrect. This has been corrected. The Erratum to this chapter is available at DOI: 10.1007/978-0-387-35620-4_43 
services over them. In order to make such services commercial, their management is of crucial importance. The management of such heterogeneous networks and services must be able to address several issues. Firstly, the integrated management of the underlying various technologies is needed. A management platform addressing in an integrated way a global network (both the radio access networks and the core network) is needed. This management platform should be able to provide both network elements (e.g. devices) and services management.

Secondly, due to the enhanced flexibility offered by SDR terminals, additional effort in the management of this flexibility must be provided. In fact, until now, the radio equipments are vertically closed, most of their configuration is done on well established and agreed parameters. Future equipments will provide more flexibility by implementing in software most functions that are performed now in hardware.

In this paper we propose the use of policy based management for the management of service and wireless networks. The integration of the management of radio resources in the more general framework of policy based management is put forward. A generic service management architecture capable to deploy policies based management on radio resources is defined. This is done by modeling the radio part and seeking to provide a coherent model for both software defined functionality and the hardware one. The main goal is to allow proprietary implementation of radio equipment to be managed in a transparent way. Since value added services are end-to-end (from the service provider to the subscriber), offered over a large variety of technology, we consider the integration of the management of radio part into a larger context of service management, especially by seeking a CIM/DEN compliance.

The paper is structured as follows: the context of the work is described in section 2 . Section 3 provides an information modeling and a management architecture for the integrated management of wireless and core network. Finally some conclusions are presented.

\section{INTEGRATED END TO END MANAGEMENT OVER HETEROGENEOUS WIRELESS DOMAINS}

Management actions are taken at several layers. Firstly, at the service level, the service manager should be able to perform some management tasks. These management actions must be next translated to radio network 
operators management actions. For the sake of clarity, only one network operator is considered in the following. At the network operators level, several management planes exist, mainly the three following ones:

- Management of the core network. When the core network is ATM or SDH based, then TMN and ATM well established paradigms can be used. In case of IP core network, IP based management can be deployed. It should be noted that the notion of IP management is becoming a real issue because of the evolution of IP networks from Best effort to QoSenabled networks. The related management has evolved from a simple devices (or network elements) management to service management.

- Management of the Radio access network. If mainly this management focuses on the radio parameters of the network elements (e.g. BTS or BSC in the case of GSM; nodeB, RNC for UMTS), the connectivity within the radio network subsystem and with the core network (the Iu, Iub, Iur interfaces in UMTS) should not be forgotten. Proprietary solutions, heavily dependent on the equipment manufacturer are currently used despite standardization effort to normalize the interface between the network elements.

- Management of the wireless terminal. Although, the potential for management is currently limited, future developments in flexible and software defined radio architecture will permit more and more configurability on the latter. Moreover, terminal management would allow the easier services management and hence facilitate the introduction of new attracting services.

One of the main challenge for the network operator is to define an integrated management architecture for the end to end services (at least, within its administrative domains), allowing to manage the three above mentioned planes by the same management architecture.

\subsection{Policy based management}

The main idea in policy based management is to deploy more intelligence onto the network entities and delegate management task to their responsibility. This is done by a set of rules consisting in a set of conditions and actions [4]. If the conditions are satisfied then the indicated actions are undertaken.

The Common Information Model/Directory Enabled Networks (CIM/DEN) [2] is an initiative supported by the IP community and is endorsed by groups like the Internet Engineering Task Force (IETF) and the Distributed Management Task Forum (DMTF) [1]. 
This initiative allows to model policies and associate them with the network entities concerned. Thus, a centralized information directory stores the policies, their relationships with other managed elements, applications and users, whilst deployment mechanisms are used to instantiate the policies on the concerned network elements. The Common Open Policy Service Protocol (COPS) [4] defines a framework for provisioning policies to network elements, based on a simple query and response protocol, used to exchange policy information between a policy server (Policy Decision Point or PDP) and its clients (Policy Enforcement Points or PEPs).

Most of current management initiatives approached the management of heterogeneous wired networks but little work has been done to integrate in these frameworks the management of wireless terminals or more generally wireless networks. A first approach towards integrated management of the radio terminal has been proposed in [8] as an over the air management of a radio terminal. This approach proposes the use of WAP as a transport layer for management information for a particular terminal technology. Recently, the MONASIDRE framework [9] proposed an interesting solution towards the management of multi-operator and multi-technologies network, without however addressing the issue of radio-terminal management. This paper will extends that work by providing a solution for an integrated management including the radio terminal part.

\section{INTEGRATED MANAGEMENT OF RECONFIGURABLE WIRELESS INFRASTRUCTURE}

The followed approach is pragmatic. We first recall the basics of the radio part of a wireless system, in order to identify what can be managed. Then an information model is derived in the larger framework of CIM/DEN initiative. Finally, a management architecture, using the information model is defined, providing integrated service and network management.

\subsection{Overview of a wireless modem}

Our interest is of course centered on digital systems since analog ones, even if some are still in use for particular applications do not meet the requirements for $3 \mathrm{G}$ and beyond $3 \mathrm{G}$ systems, especially in the convergence of data and mobile communications.

All digital wireless systems are structured into a set of basic functions as depicted in Figure 1, even if sometimes the order of the functions are 
slightly modified. It should be noted that these functions could be paired, one acting in the transmitter and a peer one in the receiver side, such as, for instance, the channel coder and the channel decoder. Generally speaking, digital streams from other users could be multiplexed at any steps in a wireless modem. The following list provides a quick overview of each pair of function. A more exhaustive functional split could be found in [5] [10].

1. Source / Recipient - AD/DA converters. The source and recipient, properly speaking can not be parametrized and thus managed: it is a picture, a voice, a movie, a file etc. However for analog source, the way the source is digitalized in the Analog to Digital Converter (ADC) could be configured thanks to the sampling speed, the number of bits of each sample. The choice of these parameters influence the bandwidth (or the data rate) needed to transmit the information as well as the quality of the signal. To keep simple, the source coder detects and reduces the inherent redundancy of a digital information.

These functions of source coding/decoding and ADC/DAC could be managed in order to control the requested bandwidth of the transmission and the perceived quality. It can be named in the extended CIM/DEM representation as a source coding service.

2. Encryption / Decryption. These functions protect the user against unwanted interception or modification of its message. Actually, in a wireless system, the transmission is made over the air. Anybody in the proximity of the radio beam could listen and try to capture the transmitted message.

A wireless operator could offer different levels of protection to different set of subscribers (basic users, VPN users) and thus needs to manage this function. Let's call it wireless encryption service.

3. Channel encoder / decoder. The channel coder consists in adding protection to the digital message in order to mitigate the harmful effect of the wireless channel. Indeed, in addition to the basic thermal noise, inherently present in all hardware equipments, a wireless channel often suffers from other disturbances: fading, multi-paths, time and frequency varying responses, interferences from other users etc.

The management actions related to channel coding belongs to the channel coding services, in the CIM/DEN description.

4. Modulator / Demodulator - Basedband / RF conversion. The next step after the protection of the digital message against the errors likely to occur on the wireless channel, is the modulation. This operation consists in transforming the bit stream into a signal that could be transmitted over the air, using radio frequencies. The message is first mapped into symbols, which are then filtered by a pulse shape filter in order to give to the signal the appropriate spectrum caracteristics. The access to the 
wireless medium of the different concurrent flows or users are then granted taken into account the requeste quality of services.

These services offered by the lowest layers of a wireless system could be thus classified as following. The bit stream services, for arbitration and multiplexing of different flows; the baseband services, covering modulation and the pulse shape filtering and the $R F$ conversion services, including the multiple access schemes as well as the management of the transmitted power.

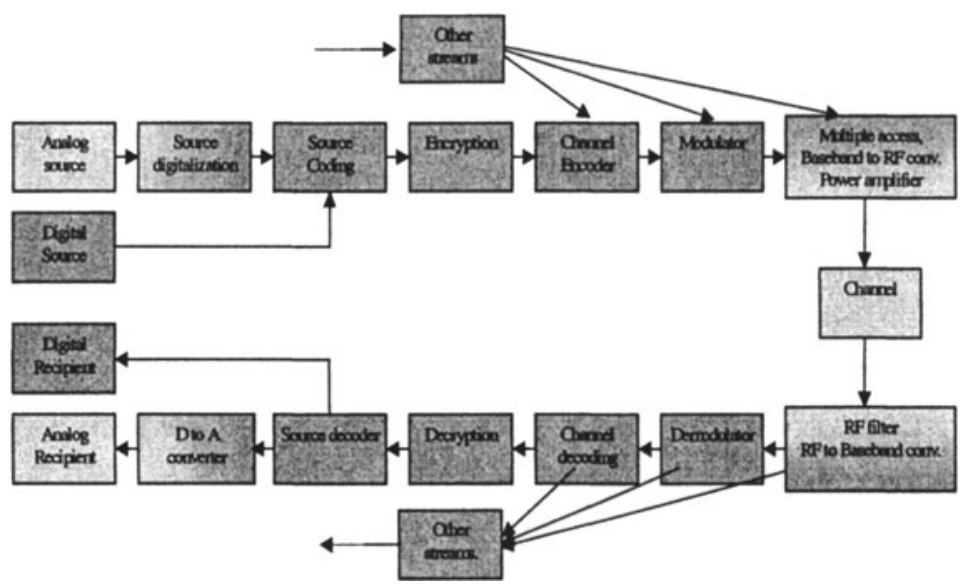

Figure 1. Architecture of a wireless modem

\subsection{Information Modeling for wireless reconfigurable infrastructures}

The information model is build based on the CIM/DEN initiative proposed by the DMTF. The CIM/DEN model allows representing management information for IP networks, system and application management.

We propose the extension of CIM through the mechanisms allowed by the DMTF in order to capture the information of the radio part in wireless networks. The goal is to allow the complete representation of such networks by integrating the model in the larger context of the CIM/DEN initiative.

The Figure 2 illustrates the proposed approach. The root of the CIM/DEN information model is the class ManagedElement. All manageable entities are modeled as descendants of this class. For instance a software installed on a system can be modeled at a lower granularity by the ServiceFeature class. An important class defined in the CIM/DEN model is 
the Service Class. We model by this class not the service itself but the its management capability. The access to a service is modeled by the ServiceAccessPoint class.

A wireless modem can provide the services previously described. In addition, a wireless device can be used as a modem only and hence could be connected to a wired network. In that case, the device should include network connection capability that could be summarized in the following as an IP Network service. Moreover, a wireless terminal could also offer a human interface, able to adapt itself according to the end user application. Such interface is captured in our model as the GUI service while the end user application is captured in the End user service. As a result, a wireless equipment could be modeled as providing the following services:

- RF Conversion service,

- Base Band service,

- Bistream service,

- Channel Coding service,

- Wireless encryption service,

- Source Coding service,

- IP Network service,

- GUI service

- End User service.

Each service is derived from the abstract class Service. As shown in the Figure 2 the service class is associated to either a Logical Device or a Software Feature. This models the way a particular service is implemented. If software defined object is used then the association SoftwareFeatureService to the Software Feature is used, else the mapping (DeviceServiceImplementation) to the Logical Device class is needed. This permits to model management of software-implemented features which is particularly useful when addressing the management of more or less software definable radio architectures. In case, hardware is used to implement these functionalities, the association to the Logical Device can be used to model the management of the hardware. Management of hardware related entities can be for instance the reconfiguration of a FPGA, the modification of a filter response or the adaptation of an antenna pattern.

Modeling the services interdependencies is done using the ServiceSAPDependency and the ServiceServiceDependency associations. For instance an instance of Bitstream Service is dependent on the Channel Coding Service. It depends also on the existence of an access point to the Channel Coding Service via the ServiceSAPDependency.

In order to illustrate how policy based management is applied using this model, the following simple scenario can be imagined. A videoconference 
service must be provided to end-users. This conference is provided by a service provider. He is not aware of the underlying existing network infrastructure but knows the user profiles and preferences. The network provider knows the status of its network, and has been informed by the service provider that at a particular time a video conference will occur between a specific set of user. Hence, he can configure its network to properly serve the delayed service request (delayed because the video conference has been booked in advance). On the user side, a simple policy could be proposed such as use WLAN if available then UMTS and finally GSM if none of the previous radio access are available. Such policy is very simple but more complex ones, ranging to dynamic spectrum rental can be also considered.

Due to the different data rates offered by WLAN, UMTS and GSM, the simple policy should be propagated through all the services provided by the terminal equipment. As an example, with UMTS the GUI offer good quality (high number of frame per second, coloured image, high quality sound) thanks to appropriate source coding service and lower level service (coding, baseband RF). In GSM only coverage, the terminal equipment must behave differently. It for instance offers only black and white jerky images with telephone like sound. We need to associate this policy with the services on the radio equipment. In this case we must change the GUI display (a software codec), if in case of a terminal equipment, change the baseband and RF service settings. These settings can be changed by either assuming the logical devices/software supporting these functionality supporting them (see the relation DeviceServiceImplementation in Figure 2) are manageable or the software implementing this functionality (relation SoftwareFeatureService) has to be downloaded. Policies can be related to a Service Level Agreement (SLA) established between users and service providers: a user might have subscribed to premium service for a video delivery service in the late evenings, whilst accepting standard quality otherwise.

This classes Setting and Configuration are used to regroup individual settings of managed objects. A setting is used to capture pre-configured parameters for one or several managed systems elements. A setting object is associated to one or several managed systems elements by the associations ElementSetting and CollectionSetting

Several setting objects can be regrouped into one configuration. Continuing with the previous example, a GSM Configuration object could regroup all individual setting objects used to configure resources needed for GSM behavior and a second object UMTS Configuration regrouping setting objects associated to UMTS type behavior. Configuration objects can be directly associated to manages systems elements. 
Several related policies are grouped in a PolicyGroup object. A policy is a rule (modeled by the class PolicyRule) consisting in a set of conditions (modeled by the class PolicyCondition) and a set of actions (modeled by the class PolicyAction). If the conditions are satisfied the indicated actions are undertaken. Such an action could be to reconfigure the equipment. We model this by the association RadioConfiguration linking the RadioPolicyAction with the RadioConfiguration Class.

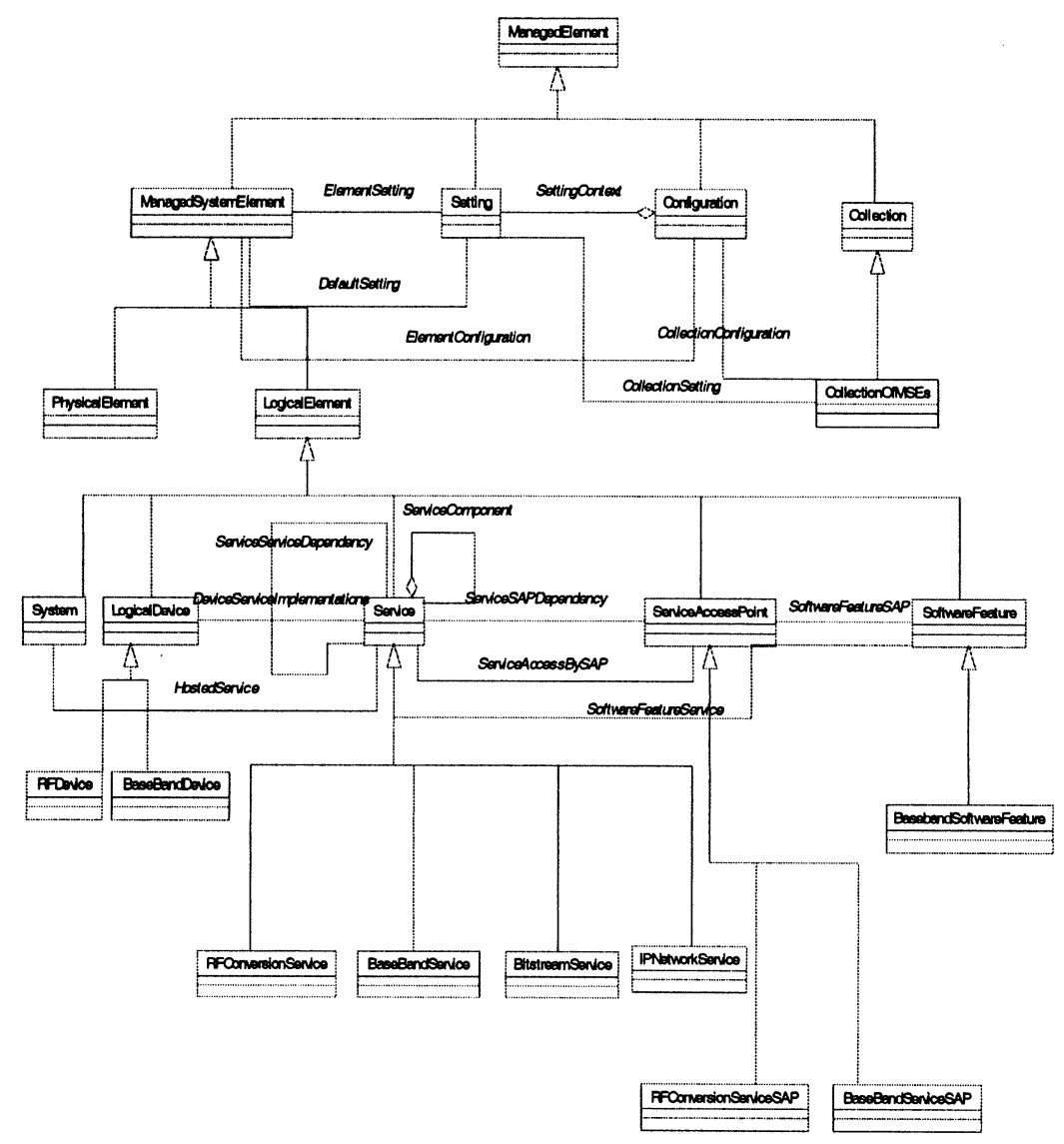

Figure 2. Model of information for a wireless system

\subsection{Management Architecture}

End-to-end service information is mapped to network level and policy level information in order to assure an integrated approach towards its 
management. The architecture is composed of different parts. We illustrate only those components concerned with policy based management. A Policy Server stores the instantiated policies. The instantiation is done with respect to the central information model. These policies are stored in a LDAP compliant server. The Policy Server is accessed over LDAP by individual PDPs which must upload their policies.

Using COPS-PR (which works over TCP connections established from the PEP to the PDP) for provisioning management entities is particular useful when this happens over error-prone and unreliable links, which are common for wireless communication.

Moreover, in some wireless networks, incoming connections to the terminal are not possible (GPRS for instance). Therefore, a communication paradigm based on a terminal initiated connectivity is particular useful and practical to allow the deployment of management function on the terminal.

Another advantage of the COPS lies in its extensibility, allowing that client or management domain specific information can be transported without requiring modifications to the COPS protocol itself.

Monitoring information is obtained through a SNMP manager. This one uses a MIB (Management Information Model) in order to obtain monitoring information. The information in the MIB is related to global information model. Finally, the common information model assures also the linking with higher level service specific management information.

One issue that must be addressed are the firewalls disrupting management traffic. In classical network management architectures, the network connectivity of the managed elements is the provided within its management domain. If we consider the case of users roaming into a foreign network and needing to be managed by their home management platform, then currently this is not possible, since the security management of the foreign network typically will disrupt TCP connections. This issue is also important when considering more advanced management scenarios in which a third party service provider could perform service specific management on the terminal 


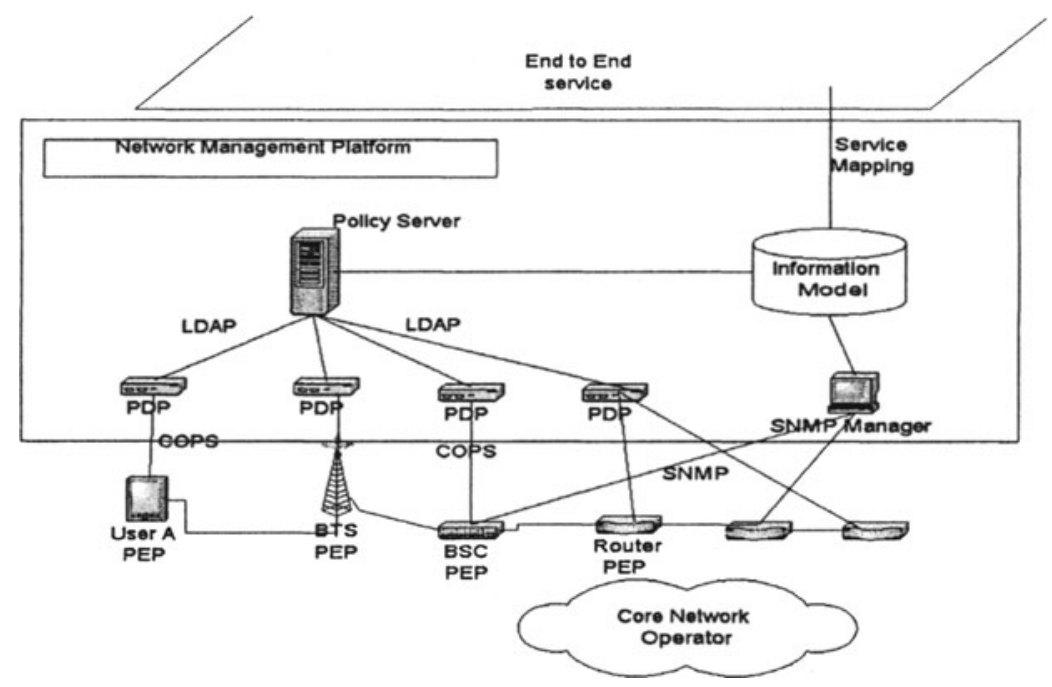

Figure 3. The management architecture

\section{CONCLUSION}

Integration of different radio access systems, convergence around IP core networks, open service architecture...These trends captured in the Beyond $3 \mathrm{G}$ vision become unavoidable and lead to the need of having unified network and service management architecture. In addition, the advent of software definable radio terminals increase the dimension of the management: terminals should be manageable too. This papers introduced a joint architecture and an information model for a joint management of this wireless world. This architecture is build on policy based management and CIM/DEN framework. Hence, this papers provides the first blocks towards an unified management of heterogeneous and reconfigurable radio networks, rather for reflection for future and deeper investigations than as an ultimate solution.

\section{REFERENCES}

[1] DMTF (www.dmtf.org) Common Information Model (CIM) Core Model

[2] J.Strassner. Directory Enabled Networks. Macmillan Technical Publishing. 1999. 
[3] W. Bumpus, A. Westerinen et al. Common Information Model: Implementing the Object Model for Enterprise Management. Wiley. 1999.

[4] D. Verma. Policy-Based Networking: Architecture and Algorithms. New Riders Publishing. 2000.

[5] J. Mitola. Software Radio Architecture. Wiley. 2000.

[6] Joint Tactical Radio System. Software Communication Architecture Specification. 2001.

[7] V. G. Bose, R. Hu, R. Morris. Dynamic Physical Layers for Wireless Networks Using Software Radio International Conference on Acoustics, Speech, and Signal Processing, Salt Lake City, UT May 2001.

[8] P. Oommen. A framework for integrated management of mobile-stations over the air. Proc of $7^{\text {th }}$. IEEE/IFIP Symposium on Integrated Management IM2001. 2001.

[9] Monasidre Project. www.monasidre.com.

[10] TRUST project. http://www.ist-trust.org 